\title{
Estágios curriculares de Odontologia nos serviços públicos de saúde após as Diretrizes Curriculares Nacionais de 2002
}

\author{
Eliana Freire dos Santos*; Fábio Barbosa de Souza**; Márcia Maria Cabral de Melo Dantas***; Sílvia \\ Regina Jamelli****; Elaine Judite de Amorim Carvalho**** \\ * Estudante, Curso de Odontologia, UFPE \\ ** Professor adjunto, Departamento de Prótese e Cirurgia Bucofacial, \\ UFPE \\ *** Professora adjunta, Departamento de Clínica e Odontologia \\ Preventiva, UFPE \\ **** Professora associada, Departamento de Clínica e Odontologia \\ Preventiva, UFPE
}

Recebido em 04/01/2018. Aprovado em 21/09/2018.

\begin{abstract}
RESUMO
O estágio curricular supervisionado nos serviços públicos de saúde, tal qual recomendado pelas Diretrizes Curriculares Nacionais (DCN), possibilita aos estudantes de Odontologia a proximidade com realidades e condutas profissionais diferentes daquelas que caracterizam sua rotina na prática clínica intramuros. O objetivo deste estudo foi realizar uma revisão da literatura sobre os estágios curriculares dos cursos de Odontologia nos serviços públicos de saúde brasileiros após as DCN publicadas em fevereiro de 2002 e assim estimular a reflexão sobre sua importância para a formação profissional contemporânea. Foi realizada uma revisão de literatura narrativa, baseada nos dados obtidos a partir da consulta em bases de dados (PubMed, SciELO, LILACS e Google Acadêmico), selecionando documentos publicados entre janeiro de 2010 e maio de 2017. A amostra final foi constituída por 15 publicações agrupadas nas seguintes categorias: relatos de experiência vivenciada no estágio curricular, percepção dos estudantes quanto ao estágio curricular e avaliação dos processos pedagógicos, técnicos e políticos na implantação do estágio. Conclui-se que os estágios curriculares das universidades brasileiras são espaços de aprofundamento no campo de trabalho que buscam aproximar os estudantes da realidade do sistema público de saúde, com intenção de formar profissionais humanitários, preocupados com o respeito e o cuidado da sociedade.
\end{abstract}

Descritores: Estágios. Educação em Odontologia. Currículo.

\section{INTRODUÇÃO}

O ensino da Odontologia tem, historicamente, se baseado na transmissão de conhecimento com foco no desenvolvimento de habilidades técnicas, nas doenças bucais e na prática clínica especializada. Um dos desafios atuais é uma formação contextualizada com a realidade e de qualidade, levando-se em 
consideração o desenvolvimento econômico, social e cultural como um novo processo de educação em saúde no Brasil. Neste contexto, as exigências das Diretrizes Curriculares Nacionais (DCN) apontam para o desafio de uma formação mais qualificada, a fim de abordar os sujeitos, famílias e comunidade no seu contexto socioeconômico e cultural ${ }^{1}$.

O Brasil implementou algumas políticas públicas de inclusão social, com o intuito de induzir o desenvolvimento do país ${ }^{2}$. Especialmente nos setores de saúde e educação foram implementadas políticas indutoras para adequar o ensino e ampliar o acesso da população à rede de serviços do Sistema Único de Saúde (SUS) de forma a responder com qualidade às necessidades $^{3}$. Na saúde há tentativas de reorganizar e estender as ações básicas, como tática de mudança do modelo centrado na doença e no atendimento especializado e complexo. $\mathrm{Na}$ educação, os debates focam as mudanças curriculares, voltadas quase exclusivamente para a eficiência e eficácia dos procedimentos, que não respondem às demandas de serviços. A relação existente entre esses dois setores diz respeito à adequação dos profissionais às necessidades sociais da população. A Odontologia se enquadra, assim, nessa nova modalidade de atenção à saúde, devendo seus profissionais cultivar uma relação também nova com a comunidade, baseada na atenção, na confiança, no respeito e no cuidado ${ }^{4}$.

Mais recentemente, como tática de mudança do modelo de atenção à saúde, optouse por implantar um modelo referenciado nos princípios da Atenção Primária à Saúde, por meio da adoção da Estratégia Saúde da Família na Política Nacional de Atenção Básica ${ }^{5}$. Na educação, os debates sobre as mudanças curriculares foram centrados com vistas a promover um perfil profissional com formação generalista, técnica, científica e humanista, com capacidade crítica e reflexiva, preparado para atuar guiado por princípios éticos, no processo de saúde-doença e em seus diferentes níveis de atenção no SUS $^{6}$.

As DCN dos Cursos de Graduação em Odontologia de fevereiro de 2002 explicitam a necessidade de metodologias que favoreçam o processo ensino-aprendizagem e a integração da universidade com os serviços de saúde, de modo a formar profissionais com ênfase na promoção de saúde e prevenção de doenças ${ }^{7}$. Nesse sentido o estágio curricular com supervisão docente, desenvolvido de forma articulada e com complexidade crescente, proporciona aos estudantes o contato com realidades e práticas profissionais diversificadas de seu cotidiano nas clínicas intramuros ${ }^{8}$.

Sob esta perspectiva de inovação pedagógica, deve-se proporcionar ao estudante, durante o processo educativo, momentos de reflexão sobre seu papel dentro de uma contextualização do seu processo de trabalho a partir da autopercepção, a fim de desenvolver, assim, potencial intelectual e afetivo que o capacite para adquirir as habilidades para atenção ao usuário do serviço de saúde e o habilite para descobrir em sua função profissional a autêntica dimensão social que cabe a ele como cidadão responsável ${ }^{9}$.

As complexidades do mundo contemporâneo aliadas aos avanços tecnológicos e científicos exigem que o conhecimento seja trabalhado, nas instituições de ensino, de forma multidimensional ${ }^{8}$. Para a formação profissional em saúde, especialmente em Odontologia, isto significa a necessidade de ampliação dos ambientes educacionais, das técnicas educativas, vivência com reais situações profissionais e com diferentes graus de complexidade.

Diante disto, realizou-se uma revisão de literatura sobre a produção recente de estudos voltados à análise dos estágios curriculares dos 
cursos de Odontologia desenvolvidos na rede de serviços de saúde públicos, após as Diretrizes Curriculares Nacionais publicadas em 2002.

\section{METODOLOGIA}

Trata-se de um estudo de revisão de literatura na modalidade narrativa. A busca de artigos foi realizada nas bases de dados PubMed, SciELO, LILACS e Google Acadêmico. A busca foi realizada utilizando as palavras-chave dental education, community outreach, community dentistry, dentistry curriculum, dental - based education.

Os títulos e os resumos de todos os artigos identificados na busca eletrônica foram lidos e analisados por duas revisoras de acordo com os seguintes critérios de inclusão: artigos publicados entre janeiro de 2010 e maio de 2017, escritos nos idiomas português, inglês ou espanhol, caracterizados como pesquisa ou estudo de caso.

A análise dos d.dos foi qualitativa. Utilizou-se uma planilha de registro de dados das variáveis: autores, ano de publicação, base de dados, ano do estudo, objetivo, abordagem metodológica quantitativa ou qualitativa e principais achados.

Posteriormente, os trabalhos foram classificados em três grupos, de acordo com o tema e a metodologia empregada: Grupo A: Relato de experiências; Grupo B: Percepção dos estudantes e Grupo C: Avaliação dos processos pedagógicos, técnicos e políticos.

\section{RESULTADOS}

Dos 15 artigos selecionados ${ }^{10-24}, 3$ foram encontrados na base de dados SciELO, 9 no Google Acadêmico, 2 no LILACS e 1 no PubMed, publicados entre os anos de 2010 e 2016 (quadro 1).

$\mathrm{O}$ quadro 2 traz as informações relativas a autores/ano e às conclusões encontradas em cada grupo. Neste formato, buscou-se apresentar de uma forma sintética a amostra selecionada nesta revisão.

Quadro 1. Distribuição dos artigos por ano de publicação, autores e base de dados encontrados

\begin{tabular}{|ccc|}
\hline Ano de Publicação & Autores & Base de dados \\
\hline 2010 & Batista et al. & SciELO \\
\hline 2011 & Warmling et al. & SciELO \\
\hline 2012 & Daher et al. & PubMed \\
\hline 2012 & Alves et al. & Google Acadêmico \\
\hline 2012 & Toassi et al. & Google Acadêmico \\
\hline 2013 & Lôbo et al. & Google Acadêmico \\
\hline 2013 & Cawahisa et al. & LILACS \\
\hline 2014 & Bulgarelli et al. & LILACS \\
\hline 2015 & Silva et al. & Google Acadêmico \\
\hline 2015 & De Melo et al. & Google Acadêmico \\
\hline 2015 & Leme et al. & Google Acadêmico \\
\hline 2015 & Gallo; Roloff & Google Acadêmico \\
\hline 2015 & Reichert et al. & Google Acadêmico \\
\hline 2015 & Junior et al. & SciELO \\
\hline 2016 & Nobrega et al. & Google Acadêmico \\
\hline
\end{tabular}


Quadro 2. Distribuição dos artigos de acordo com os grupos, autores, ano de publicação e conclusões

\begin{tabular}{|c|c|c|}
\hline Grupo & Autores/Ano & Conclusão \\
\hline \multirow{5}{*}{ A } & Nobrega et al., 2016 & Potencializa o conhecimento do território e dos problemas da comunidade e do próprio serviço de saúde \\
\hline & Silva et al., 2015 & $\begin{array}{l}\text { Possibilita além da ocorrência de práticas pedagógicas nos cenários de práticas do SUS, também o } \\
\text { aprendizado de competências e habilidades descritas pelos DCN }\end{array}$ \\
\hline & Gallo; Roloff, 2015 & $\begin{array}{l}\text { Constrói uma aprendizagem do funcionamento das redes de atenção especializada em saúde bucal e } \\
\text { aperfeiçoar competências para que haja a resolutividade dos obstáculos }\end{array}$ \\
\hline & Cawahisa et al., 2013 & $\begin{array}{l}\text { Propicia atividades que se validam na criação de novas práticas de saúde, com uma relação de trabalho } \\
\text { baseada na atuação multiprofissional, visando à promoção da saúde da comunidade }\end{array}$ \\
\hline & Daher et al., 2012 & $\begin{array}{l}\text { Primeira impressão positiva após uma curta estadia em um programa de serviço comunitário, mas houve } \\
\text { uma diminuição nas experiências positivas dos alunos ao longo do tempo }\end{array}$ \\
\hline \multirow{4}{*}{ B } & Batista et al., 2010 & Proporciona a compreensão dos alunos sobre o funcionamento do SUS \\
\hline & Reichert et al., 2015 & $\begin{array}{l}\text { Compreendem a importância dos estágios supervisionados para a sua formação acadêmica, uma vez que } \\
\text { estes permitem maior aproximação com a população, o conhecimento e a vivência do SUS }\end{array}$ \\
\hline & Bulgarelli et al., 2014 & $\begin{array}{l}\text { Percebem diferentes processos de trabalho em saúde, apontam a importância do engajamento dos } \\
\text { professores na supervisão dos estágios e percebem o SUS como um rico espaço de aprendizagem }\end{array}$ \\
\hline & Lôbo et al., 2013 & $\begin{array}{l}\text { Compreendem que a pesar das deficiências relatadas sobre a infraestrutura dos estágios eles contribuíram } \\
\text { para a formação do egresso, por propiciarem uma aproximação com a realidade social e dos serviços de } \\
\text { saúde }\end{array}$ \\
\hline $\mathrm{C}$ & Warmling et al., 2011 & $\begin{array}{l}\text { A integração do ensino no serviço é eixo fundamental dos processos de mudanças que vem ocorrendo tanto } \\
\text { no ensino superior como nos modelos e práticas públicos }\end{array}$ \\
\hline
\end{tabular}

Grupo A: Relato de experiências; Grupo B: Percepção dos estudantes; Grupo C: Avaliação dos processos pedagógicos, técnicos e políticos 


\section{DISCUSSÃO}

A maioria dos artigos selecionados foram publicados entre 2012 e 2015. Constatou-se que os artigos começaram a ser disponibilizados apenas quase uma década depois da promulgação das DCN, embora as instituições de ensino superior (IES) tenham inserido práticas de estágios curriculares supervisionados logo após ou até mesmo antes das DCN serem instituídas ${ }^{25}$. Este fato pode ser justificado pela necessidade de acúmulo necessário de experiências, o que demandou a espera pela conclusão das turmas que iniciaram o curso após a mudança curricular.

De modo geral, as situações dos estágios revelam variantes positivas que contribuem para a consolidação dos objetivos propostos pelas DCN. Porém, nessas mesmas experiências, existem dificuldades e problemáticas a serem superadas, tais como o pouco engajamento e a incompreensão dos docentes em relação ao funcionamento do SUS $^{11,17}$, bem como a indefinição do papel do preceptor e a pouca expansão da Atenção Básica ${ }^{11}$.

Como se observa no quadro 1, a maioria dos artigos relatou experiências exitosas com ampliação dos conhecimentos e práticas dos estudantes, além de maior compreensão da realidade vivenciada no SUS. Esses resultados são observados em um estudo no qual os objetivos propostos foram atingidos pela integração ensino-serviço na USF, enquanto processo que potencializa a formação do profissional voltado para atuar no SUS e com perfil adequado às reais necessidades da população brasileira $^{13}$.

As atividades curativas e educativas realizadas pelos acadêmicos no estágio possibilitaram o desenvolvimento das seis competências e habilidades gerais requeridas para a formação do cirurgião-dentista propostas pelas DCN: atenção à saúde, tomada de decisões, comunicação, liderança, administração e gerenciamento e educação permanente ${ }^{15}$.

Os graduandos, por meio da participação em palestras, visitas domiciliares, atividades realizadas na pastoral, na creche, nas escolas e na própria USF puderam entrar em contato íntimo e amplo com a realidade da atuação de um profissional na área da saúde pública ${ }^{10}$.

Ao inserir o estudante no serviço, ele passa a atuar com o cirurgião-dentista (CD) trabalhador do SUS em uma equipe multiprofissional e de forma interdisciplinar. O profissional que assume o papel de preceptor do estudante não pertencente ao corpo docente da IES, sendo responsável pela inserção e socialização do estudante no ambiente de trabalho, estreitando, assim, a distância entre teoria e trabalho. Este foi um aspecto positivo observado em pesquisa sobre a satisfação dos alunos com este componente curricular ${ }^{26}$

A relação dos estudantes com os CDs preceptores introduz o interesse pelo serviço público de saúde, e, de certo modo, a vivência no estágio foi capaz de mudar os paradigmas de percepção tecnicista da profissão ${ }^{17,24}$.

Contudo, foram identificadas dificuldades com relação ao planejamento dos estágios, com sugestão de reuniões prévias para que sejam esclarecidas e pactuadas no início das atividades as atribuições dos estudantes e supervisores de estágio. Essa percepção foi mais evidente para estudantes da metade e do final do curso, uma vez que têm um maior contato individual com os profissionais da rede ${ }^{22}$.

Entre outras dificuldades apresentadas, a resistência por parte de alguns alunos em se inserir de forma "obrigatória" (curricular) em unidades do SUS, alegando falta de interesse para atuar no serviço público como futuro profissional, falta de segurança e dificuldade de deslocamento. Entretanto, a experiência relatada 
pelos estagiários das primeiras turmas vem demonstrando aos alunos das turmas subsequentes a importância do estágio na formação acadêmica e profissional. Infelizmente, esse ganho de qualidade na formação não é compreendido por alguns professores que acreditam que os alunos formandos seriam melhor preparados nas clínicas integradas intramuros ${ }^{23}$.

Problemas relacionados à falta de material, de infraestrutura e estrutura para que a carga horária possa ser cumprida da maneira correta foram também observadas por outros autores ${ }^{21,24}$, fato ressaltado em um estudo que supõe existir uma grande deficiência na organização dos serviços públicos de saúde, trazida por dificuldade de financiamento ou de gerenciamentos dos seus recursos. Apesar disso, a falta de material e equipamentos não trouxe maior prejuízo no comprimento do cronograma, mas gerou necessidade de adaptação das técnicas e dos materiais utilizados, acarretando resultados menos satisfatórios ${ }^{15}$.

De acordo com as diretrizes da Associação Brasileira de Ensino Odontológico (ABENO) para a definição do estágio supervisionado curricular, os estágios devem possibilitar ao estudante o reconhecimento da realidade social, do funcionamento dos serviços de saúde, articular a teoria com a prática em situação real e cotidiana, conhecer a realidade do mercado de trabalho e se apropriar da lógica do sistema de saúde, em todas as suas dimensões ${ }^{27}$.

Conforme pode ser observado no quadro 2 , a experiência no estágio proporcionou aos acadêmicos o desvelamento da realidade social e pareceu colaborar com a construção de uma visão humanista e compreensiva dos problemas de saúde $^{20}$. Realmente a interação entre ensinoserviço-comunidade proporcionou crescimento dos estudantes, permitindo-os vivenciar as reais necessidades da comunidade na qual está inserida $^{13}$.

Ainda confirmando a contribuição dos estágios no SUS para a formação de um novo perfil de profissional, esta experiência constrói percepções de interesse dos estudantes para uma atuação na saúde coletiva. De certo modo, o despertar desse interesse reflete a futura atuação de CDs bem preparados para o trabalho no serviço público de saúde. Existe o reconhecimento do estudante de que, sem a participação do SUS na sua formação, não teria construído uma compreensão sólida do funcionamento e dos conceitos do sistema público de saúde para a possibilidade de futura atuação no sistema público ${ }^{17}$.

A expectativa de vivenciar novas e inexploradas situações, compreender a dinâmica do trabalho em uma unidade de saúde e os sentimentos de insegurança inicial bem como de surpresa frente a realidade que desconheciam ficam marcados nos relatos dos estudantes, assim como o sentimento de superação diante das expectativas preliminares ${ }^{14}$.

Nessa perspectiva, observando-se o quadro 2, pode-se afirmar que a percepção dos estudantes quanto ao estágio curricular revelou que as práticas extramurais oferecem a oportunidade para que vivenciem o funcionamento do SUS, proporcionando a formação de profissionais mais humanistas e com consciência social.

O real cumprimento das DCN implica uma verdadeira mudança da graduação ${ }^{28}$. Faz-se necessária a avaliação permanente da experiência e dos rumos de cada instituição ${ }^{29}$. Essas opiniões vão ao encontro de afirmações de um estudo reproduzidas no quadro 2 , em que se alega que para serem possíveis os processos de mudanças no ensino superior e nos modelos e práticas públicos, torna-se necessário analisar e divulgar como essas experiências vêm sendo desenvolvidas, seus embates e suas conquistas ${ }^{11}$. 


\section{CONCLUSÃO}

Existem relatos de várias experiências exitosas de estágios curriculares de Odontologia no SUS. Contudo, persistem desafios a serem superados em relação à sua organização, planejamento e implantação. Os estágios supervisionados no sistema público de saúde brasileiro se encontram em um processo de construção, porém já constituem um avanço expressivo no contato dos estudantes com a sociedade e suas reais necessidades, favorecendo o a formação de profissionais como instrumentos de promoção e a prevenção da saúde.

\section{ABSTRACT \\ Dentistry curricular internship in the public health services after the 2002's National Curricular Guidelines}

The supervised curricular internship in the public health services, as recommended by the National Curricular Guidelines (DCN), allows dentistry leads to dentistry students the proximity to the realities and professional conduct different from those performed in their routine searching for teaching-service's health integration. The objective of this work was to conduct a review of the literature on the curricular internship in the public health service of dentistry courses of Brazilian's Higher Education Institutions after the new DCN on February 2002 and thus encourage the reflection of its value for the contemporary professional formation. A review of narrative literature was made out, based on the data obtained from the electronic databases (PubMed, SciELO, LILACS and Google Acadêmico) published between January 2010 and Mayo 2017. The final sample was consisted of 15 grouped as following: Reports of the experience lived in the Curricular Internship, students' perceptions of the curricular internship and evaluation of the pedagogical technical and political processes in the implantation of the Curricular Internship. In conclusion, the curricular internships conducted nowadays in the Brazilians Universities are points to go further in the work field, which seeks to bring students closer to the reality of the current health public system, intending to train humanitarian professionals, concerned with society's respect and care.

Descriptors: Curricular Internship. Education, Dental. Curriculum.

\section{REFERÊNCIAS}

1. Forte FDS, Pessoa TRRF, Freitas CHSM, Pereira CAL, Junior PMC. Reorientação na formação de cirurgiões-dentistas. Interface Comum Saúde Educação. 2015;19(1):83143.

2. Paim JS. A constituição cidadã e os 25 anos do sistema único de saúde (SUS). Cad Saúde Pública. 2013;29(10):1927-53.

3. Feuerwerker L, Almeida M. Diretrizes curriculares e projetos pedagógicos: é tempo de ação. Rev ABENO. 2004;4(1):14-6.

4. Villalba JP. Perfil profissional dos cirurgiões-dentistas e o sistema único de saúde: uma reflexão sobre a capacitação para a prática na atenção básica [tese]. Campinas: Universidade Estadual de Campinas; 2007.

5. Macinko J, Harris MJ. Brazil's family health strategy -delivering community-based primary care in a universal health system. $\mathrm{N}$ Engl J Med. 2015;372:2177-81.

6. Lopes Neto D, Teixeira E, Vale EG, Cunha FS, Xavier IM, Fernandes JD, et al. Aderência dos cursos de graduação em enfermagem às diretrizes curriculares nacionais. Rev Bras Enferm. 2007; 60(6): 627-34.

7. Moura ELDS, de Oliveira EEG, Safh F, do Nascimento 1S, Brandão GAM. Práticas de odontologia em saúde coletiva na estratégia de saúde da família. Rev ABENO. 2016;15(3):52-9.

8. Fonseca EP. As diretrizes curriculares nacionais e a formação do cirurgião-dentista brasileiro. J Manag Prim Health Care. 2013;3(2):158-78. 
9. Gonçalves PE, Gabin CAS. A promoção de saúde no ensino odontológico. Rev Ciênc Méd. 2016;24(2):55-61.

10. Batista MJ, Gibilini C, Kobayashi HM, Ferreira LL, Gonçalo CDS, Sousa MDLRD. Relato de experiência da interação entre universidade, comunidade e unidade de saúde da família em Piracicaba, SP, Brasil. Arq Odontol. 2010;46(3):144-51.

11. Warmling CM, Rossoni E, Hugo FN, Toassi RFC, Lemos VAD, Slavutzki SMBD, Rosa ARD. Estágios curriculares no SUS: experiências da Faculdade de Odontologia da UFRGS. Rev ABENO. 2011;11(2):6370.

12. Daher MAS, Luciane R, Costa PHD, Geovanna CM, Machado DDS. Dental students' perceptions of community-based education: A retrospective study at a dental school in Brazil. J Dent Educ. 2012;76(9):1218-25.

13. Alves LA, De Almeida Freires I, Braga CC, De Castro RD. Integração ensino-serviço: experiência exitosa na atenção odontológica à comunidade. Rev Bras Ci Saúde. 2012;16(2):235-8.

14. Toassi RFC, Davoglio RS, Lemos VMA. Integração ensino-serviço-comunidade: o estágio na atenção básica da graduação em Odontologia. Educ Rev. 2012;28(4):223-42.

15. Lôbo AEDA. Estágios curriculares: óptica do egresso do curso de odontologia da Universidade Federal do Ceará [dissertação]. Fortaleza: Universidade Federal do Ceará; 2013.

16. Cawahisa PT, Terada RSS, Hayacibara MF. Atividades realizadas durante o estágio supervisionado do curso de odontologia da UEM em um centro de educação infantil. Ciênc Cuid Saúde. 2013;12(2):37581.

17. Bulgarelli AF, Souza KR, Baumgarten A,
Souza JM, Rosing CK, Toassi RFC. Formação em saúde com vivência no Sistema Único de Saúde (SUS): percepções de estudantes do curso de Odontologia da Universidade Federal do Rio Grande do Sul (UFRGS). 2014;18(49):351-62.

18. Da Silva MA, Mendes AAJ, Ferreira EF. La importancia de las etapas curriculares supervisadas en el sistema único de salud (SUS) para la formación académica brasileña y la enseñanza odontológica. Educ Méd Sup. 2015;29(3):617-33.

19. Melo MMDC, Jamelli SR, Carvalho EJA, SILVA CHV, Góes PSA, Souza FB, Figueiredo N. Os estágios curriculares em Odontologia na atenção primária à saúde do Recife, Pernambuco, Brasil. In: Convención Internacional de Salud, Cuba Salud 2015. Abril de 2015. Palacio de las Convenciones. Havana - Cuba: eCIMED; 2015.

20. Leme PAT, Pereira AC, Meneghim MC, Mialhe FL. Perspectivas de graduandos em odontologia acerca das experiências na atenção básica para sua formação em saúde. Ciênc Saúde Coletiva. 2015;20(4):1255-65.

21. Gallo DB, Roloff JR. Estágio curricular supervisionado em serviços de gestão e de atenção especializada em saúde bucal do Sistema Único de Saúde, 2013 a 2015 [trabalho de conclusão de curso]. Porto Alegre: Universidade Federal do Rio Grande do Sul; 2015.

22. Reicher A, Pessoa T, Forte F. Significado dos estágios supervisionados para estudantes de odontologia. Atas CIAIQ. 2015;1:251-4.

23. Junior S, Ferreira M, Pacheco KTDS, de Carvalho RB. Multiplicidade de atuações do acadêmico de Odontologia no estágio curricular: relato de experiência. Arq Odontol. 2015;51(4)194-204.

24. Nobrega DR. O estágio na atenção primária 
à saúde como ferramenta potencializadora na formação do cirurgião-dentista: Um relato de experiência [trabalho de conclusão de curso]. Araruna: Universidade Estadual da Paraíba; 2016.

25. Fonsêca GS, Junqueira SR, de Araújo ME, Botazzo C. Modelo lógico-ideal para o estágio curricular supervisionado: a educação pelo trabalho na formação Odontológica. Rev ABENO. 2015;15(2):211.

26. da Luz GW, Toassi RFC. Percepções sobre o preceptor cirurgião-dentista da Atenção Primária à Saúde no ensino da Odontologia. Rev ABENO. 2016;16(1):2-12.

27. Scavuzzi AIF, de Gouveia CVD, Carcereri DL, Veeck EB, Ranali J, da Costa LJ, de Araújo ME. Revisão das Diretrizes da ABENO para a definição do Estágio Supervisionado Curricular nos cursos de Odontologia. Rev ABENO. 2016;15(3):109-
13.

28. Moreira COF, de Araújo Dias MS. Diretrizes curriculares na saúde e as mudanças nos modelos de saúde e de educação. ABCS Health Sciences. 2015;40:(3):300-5.

29. Toassi RFC, Souza JMD, Baumgarten A, Rösing CK. Avaliação curricular na educação superior em odontologia: discutindo as mudanças curriculares na formação em saúde no Brasil. Rev ABENO. 2012;12(2):170-7.

\section{Correspondência para:}

Elaine Judite de Amorim Carvalho e-mail: elaine judite@yahoo.es

Rua Quimera. Q-D4. N²9

53370-290 Olinda/PE 